\title{
Расчет температурной зависимости энергии состояний кулоновского акцептора в узкозонном твердом растворе $\mathrm{HgCdTe}$
}

\author{
(C) М.С. Жолудев ${ }^{1,2}$, В.В. Румянцев ${ }^{1,2}$, С.В. Морозов ${ }^{1,2}$ \\ ${ }^{1}$ Институт фризики микроструктур Российской академии наук, \\ 603950 Нижний Новгород, Россия \\ ${ }^{2}$ Нижегородский государственный университет им. Н.И. Лобачевского, \\ 603950 Нижний Новгород, Россия \\ E-mail: zholudev@ipmras.ru
}

Поступила в Редакцию 12 апреля 2021 г.

В окончательной редакции 19 апреля 2021 г.

Принята к публикации 19 апреля 2021 г.

\begin{abstract}
Проведены расчеты энергий локализованных и резонансных состояний кулоновского акцептора в узкозонном твердом растворе $\mathrm{HgCdTe}$ в зависимости от температуры. Предложен универсальный метод вычисления энергий локализованных и резонансных примесных состояний. Теоретически доказано, что эти энергии практически не изменяются в диапазоне температур от 0 до $100 \mathrm{~K}$. Расчеты проведены в приближении сферической симметрии с помощью метода матрицы рассеяния в рамках трехзонной модели Кейна, учитывающей зону проводимости и две верхние валентные зоны.
\end{abstract}

Ключевые слова: примесь, резонансные состояния, кадмий-ртуть-теллур, матрица рассеяния.

DOI: $10.21883 /$ FTP.2021.10.51434.33

\section{1. Введение}

Тройное соединение кадмий-ртуть-теллур (КРТ) на протяжении нескольких десятилетий является объектом как фундаментальных, так и прикладных исследований. Причина постоянного интереса к этому материалу особенности зонной структуры CdTe и $\mathrm{HgTe}$. Полупроводник CdTe обладает нормальной зонной структурой, при которой зона проводимости образована волновыми функциями с симметрией $\Gamma_{6}$, а валентная зона - волновыми функциями с симметрией $\Gamma_{8}$, и при низких температурах ширина запрещенной зоны в нем составляет $\sim 1.6$ эВ. В то же время благодаря более сильному спинорбитальному взаимодействию $\mathrm{HgTe} \mathrm{имеет} \mathrm{инвертиро-}$ ванную зонную структуру. Здесь и зона проводимости, и валентная зона имеют симметрию $\Gamma_{8}$ и касаются друг друга, а зона с симметрией $\Gamma_{6}$ располагается на $\sim 0.3$ эВ ниже точки касания. Таким образом, данный материал является бесщелевым, и иногда говорят, что ширина запрещенной зоны в нем отрицательна, имея при этом в виду величину $E_{g}=E_{c}-E_{v}[1]$. В твердых растворах $\mathrm{Cd}_{x} \mathrm{Hg}_{1-x}$ Tе значение этой величины зависит от состава $x$ и может варьироваться в широких пределах: от -0.3 до 0 эВ при инвертированной зонной структуре и от 0 до 1.6 эВ при нормальной зонной структуре.

Недавние успехи в технологии изготовления гетероструктур с квантовыми ямами $\mathrm{Hg}_{1-x} \mathrm{Cd}_{x} \mathrm{Te} / \mathrm{Cd}_{y} \mathrm{Hg}_{1-y} \mathrm{Te}[2,3]$ и получении в них стимулированного излучения в дальнем инфракрасном диапазоне [4] открывают возможности для разработки новых оптоэлектронных приборов на основе данного материала. Необходимость создания $p-n$-переходов, а также неизбежное присутствие в данном полупроводнике остаточных примесей и дефектов делает весьма актуальной задачу изучения электрических и оптических свойств таких центров. В последнее время были достигнуты некоторые успехи в теоретическом описании дискретного спектра примесей в КРТ, в том числе наиболее распространенных из них - вакансий ртути, которые являются двухзарядными акцепторами [5,6]. В то же время в узкозонных образцах, когда ширина запрещенной зоны оказывается меньше или равна характерной энергии примесных уровней, следует ожидать возникновения резонансных состояний. Спектральные линии, свидетельствующие о наличии таких состояний, уже были обнаружены в ходе экспериментов [7], где было продемонстрировано, что их положение не изменяется, несмотря на значительный сдвиг положения зоны проводимости при изменении температуры. Однако теоретические оценки их энергии были основаны на модели Латтинжера, не учитывающей зону проводимости [7]. Так как резонансные уровни располагаются непосредственно в этой зоне, количественная оценка ее влияния представляется необходимой для объяснения природы спектральных особенностей, наблюдавшихся в работе [7]. В то же время расчет примесных состояний в непрерывном спектре связан с рядом сложностей, главная из которых - сильная неустойчивость уравнения для их волновой функции и невозможность найти его решение прямыми методами [8].

В данной работе мы предлагаем новый подход к теоретическому описанию примесных состояний в узкозонных полупроводниках, основанный на методе матрицы рассеяния. Будет показано, что данный метод позволяет не только вычислить волновые функции непрерывного спектра, но также определить энергии локализованных и резонансных состояний. В качестве примесного центра 
мы рассмотрим кулоновский акцептор в узкозонном соединении $\mathrm{Hg}_{0.84} \mathrm{Cd}_{0.16}$ Те. Материал выбран таким, чтобы при изменении температуры его зонная структура испытывала не только количественные (ширина запрещенной зоны), но и качественные изменения (переход от инвертированной зонной структуры к нормальной).

\section{2. Метод расчета}

Энергия и волновая функция примесного состояния являются решениями стационарного уравнения Шредингера:

$$
\left(\hat{H}_{0}+V(r)\right) \psi(\mathbf{r})=E \psi(\mathbf{r})
$$

где $\hat{H}_{0}$ - гамильтониан электрона в однородном полупроводнике, а $V(r)$ - потенциал примеси. Мы воспользуемся приближением огибающих функций и будем считать нашу систему сферически симметричной. Тогда базисные функции $u_{S, S_{z}}(\mathbf{r})$ будут иметь определенные значения полного углового момента $S$ и его проекции $S_{z}[9,10]$.

Так как в узкозонном КРТ зона с симметрией $\Gamma_{6}$ лежит к зонам $\Gamma_{8}$ значительно ближе, чем спин-отщепленная зона, мы будем использовать трехзонную модель Кейна со следующим набором базисных функций:

$$
\Gamma_{6}: u_{1 / 2, \pm 1 / 2}, \quad \Gamma_{8}: u_{3 / 2, \pm 1 / 2}, u_{3 / 2, \pm 3 / 2}
$$

В рамках приближения огибающих функций, на основе данного базиса мы можем построить собственные функции полного углового момента с квантовым числом $J$ и его проекции на ось $z$ с квантовым числом $M$ :

$$
\Phi_{L, S}^{(J, M)}(\mathbf{r})=\sum_{L_{z}, S_{z}} C_{L, L_{z} ; S, S_{z}}^{J, M} Y_{L, L_{z}}(\theta, \varphi) u_{S, S_{z}}(\mathbf{r}),
$$

где $L=J \pm 1 / 2, S=1 / 2,3 / 2$, а $C_{L, L_{z} ; S, S_{z}}^{J, M}-$ коэффициенты Клебша-Гордона. Общее решение уравнения (1) будет линейной комбинацией таких функций с коэффициентами, зависящими от расстояния до примесного центра $(r)$. Такую функцию можно представить в виде произведения вектора-строки из базисных функций (2) на неизвестный вектор-столбец $\mathbf{f}^{(J, L)}(r)$ :

$$
\psi_{J, L, M}(\mathbf{r})=\left(\begin{array}{lll}
\Phi_{L, 1 / 2}^{(J, M)} & \Phi_{L+1,3 / 2}^{(J, M)} & \Phi_{L-1,3 / 2}^{(J, M)}
\end{array}\right)\left(\begin{array}{c}
f_{1}^{(J, L)}(r) \\
f_{2}^{(J, L)}(r) \\
f_{3}^{(J, L)}(r)
\end{array}\right)
$$

Подставив выражение (3) в уравнение Шредингера (1), мы можем получить [8-10] уравнение для вектора $\mathbf{f}(r)$ следующего вида:

$$
\left(\hat{\mathbf{H}}_{0}(J, L)+V(r)\right) \mathbf{f}(r)=E \mathbf{f}(r)
$$

где гамильтониан электрона в однородном полупроводнике теперь представляет собой матрицу операторов:

$$
\begin{aligned}
& \hat{H}_{0}(J, L)= \\
& =\left(\begin{array}{ccc}
E_{g}+A_{c} \hat{K}_{-}^{(L+1)} \hat{K}_{+}^{(L)} & P_{-} \hat{K}_{-}^{(L+1)} & P_{+} \hat{K}_{+}^{(L-1)} \\
P_{-} \hat{K}_{+}^{(L)} & -\gamma_{+} \hat{K}_{-}^{(L+2)} \hat{K}_{+}^{(L+1)} & -v_{2} \hat{K}_{+}^{(L)} \hat{K}_{+}^{(L-1)} \\
P_{+} \hat{K}_{-}^{(L)} & -v_{2} \hat{K}_{-}^{(L)} \hat{K}_{-}^{(L+1)} & -\gamma_{-} \hat{K}_{-}^{(L)} \hat{K}_{+}^{(L-1)}
\end{array}\right),
\end{aligned}
$$

в которой использованы следующие обозначения:

$$
\begin{aligned}
& \hat{K}_{+}^{(L)}=-\frac{\partial}{\partial r}+\frac{L}{r} \\
& \hat{K}_{-}^{(L)}=\frac{\partial}{\partial r}+\frac{L+1}{r}, \\
& A_{c}=\frac{\hbar^{2}}{2 m_{0}}(1+2 F)+\frac{P^{2}}{3\left(E_{g}+\Delta\right)}, \\
& P_{+}=P w_{+}(J, L), \\
& P_{-}=P w_{-}(J, L), \\
& \gamma_{ \pm}(J, L)=\frac{\hbar^{2}}{2 m_{0}}\left(\gamma_{1} \pm \frac{4 \gamma_{2}+6 \gamma_{3}}{5} w(J, L)\right) \text {, } \\
& \nu_{2}(J, L)=\frac{\hbar^{2}}{2 m_{0}} \frac{4 \gamma_{2}+6 \gamma_{3}}{5} w_{2}(J, L), \\
& w(J, L)=-3 / 2\left(w_{+}^{2}(J, L)-w_{-}^{2}(J, L)\right), \\
& w_{2}(J, L)=3 w_{+}(J, L) w_{-}(J, L), \\
& w_{+}(J, J-1 / 2)=\sqrt{(2 J-1) / 4 J}, \\
& w_{-}(J, J-1 / 2)=\sqrt{(2 J+3) / 12 J}, \\
& w_{+}(J, J+1 / 2)=\sqrt{(2 J-1) / 4(J+1)}, \\
& w_{-}(J, J+1 / 2)=\sqrt{(2 J+3) / 12(J+1)} .
\end{aligned}
$$

Таким образом, мы получили систему обыкновенных дифференциальных уравнений для радиальных функций $\mathbf{f}(r)$. Здесь необходимо отметить, что при $J=1 / 2$ следует положить $f_{3}(r) \equiv 0$ и отбросить последние строку и столбец гамильтониана, так как $\Phi_{L \leq 0,3 / 2}^{(1,2, M)}(\mathbf{r}) \equiv 0$.

Рассмотрим однородный полупроводник, т. е. положим $V(r)=0$. В этом случае общее решение уравнения (4) имеет вид

$$
\mathbf{f}(r)=\left(\begin{array}{c}
c_{1} j_{L}(k r) \\
c_{2} j_{L+1}(k r) \\
c_{3} j_{L-1}(k r)
\end{array}\right)
$$

где $j_{L}-$ сферические функции Бесселя. В отличие от нашей предыдущей работы [8], здесь операторы $\hat{K}_{ \pm}^{(L)}$ определены таким образом, чтобы их действие на данные функции давало следующий результат:

$$
\begin{aligned}
& \hat{K}_{+}^{(L)} j_{L}(k r)=k j_{L+1}(k r), \\
& \hat{K}_{-}^{(L)} j_{L}(k r)=k j_{L-1}(k r) .
\end{aligned}
$$




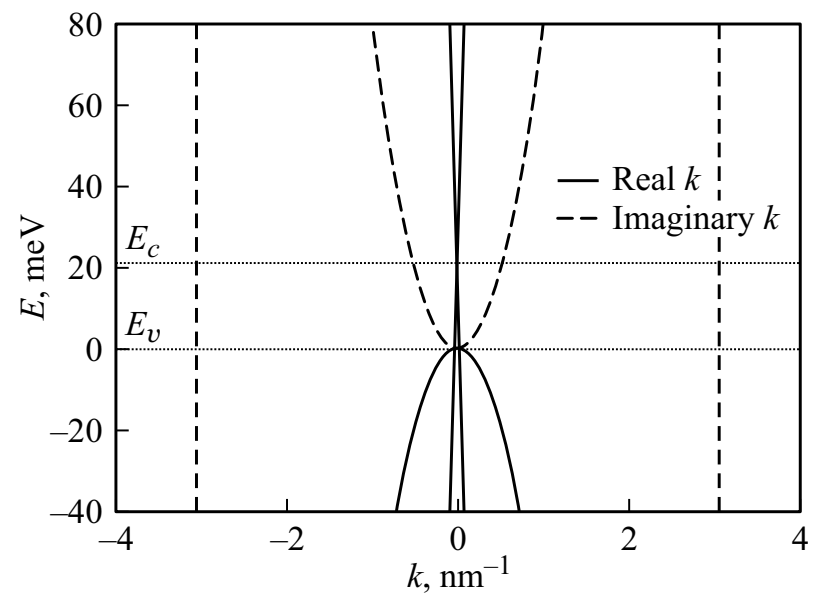

Рис. 1. Закон дисперсии для объемного $\mathrm{Hg}_{0.82} \mathrm{Cd}_{0.18} \mathrm{Te}$ в приближении сферической симметрии.

Подставив решение (5) в уравнение (4) и воспользовавшись выражениями (6), мы получим систему линейных уравнений:

$$
\mathbf{H}(k) \mathbf{c}=E \mathbf{c},
$$

где

$$
\begin{gathered}
\mathbf{H}(k)=\left(\begin{array}{ccc}
E_{g}+A_{c} k^{2} & P_{-} k & P_{+} k \\
P_{-} k & -\gamma_{+} k^{2} & -v_{2} k^{2} \\
P_{+} k & -v_{2} k^{2} & -\gamma_{-} k^{2}
\end{array}\right), \\
\mathbf{c}=\left(\begin{array}{c}
c_{1} \\
c_{2} \\
c_{3}
\end{array}\right) .
\end{gathered}
$$

Ненулевые решения уравнения (7) существуют только при условии

$$
\operatorname{det}(\mathbf{H}(k)-E)=0 .
$$

Это выражение определяет зависимость $E(k)$, т. е. закон дисперсии для однородного полупроводника.

При каждом значении $E$ уравнение (8) имеет 6 решений для $k$. Характерный вид этих решений (как действительных, так и мнимых) для узкозонного полупроводника показан на рис. 1. Нетрудно заметить, что при любых значениях энергии существует, как минимум, две ветки мнимых решений с очень большими по модулю значениями $\left(\sim 3 \mathrm{Hм}^{-1}\right)$. При отрицательных мнимых $k$ функции $j_{L}(k r)$ в выражении (5) будут очень быстро экспоненциально расти. Наличие таких решений приводит к сильной неустойчивости, из-за которой вычисление радиальных функций путем прямого интегрирования дифференциальных уравнений (4) не представляется возможным.

Аналогичная проблема возникает в задачах туннелирования через большое количество высоких барьеров (или при учете нескольких долин) [11]. В этом случае удобно использовать метод матрицы рассеяния, который благодаря явному разделению растущих и убывающих решений позволяет избежать неустойчивости. При использовании этого подхода все экспоненциальные решения фактически являются убывающими (подробнее см. работу [11]).

Данный метод уже применялся для решения уравнений с непрерывно меняющимся потенциалом [12-14], в том числе и в наших предыдущих работах для модели Латтинжера $[15,16]$. Для этого сначала ось координат разбивается на отрезки, на каждом из которых вычисляются все частные решения уравнения (4). В нашем случае на отрезке $\left[r_{n}, r_{n+1}\right]$ существует ровно 6 линейно независимых решений $\mathbf{f}_{n}(r)$, которые нужно сгруппировать в 2 матрицы по 3 столбца:

$$
\begin{aligned}
& \mathbf{F}_{n}^{(a)}(r)=\left(\mathbf{f}_{n, 1}^{(a)}(r) \mathbf{f}_{n, 2}^{(a)}(r) \mathbf{f}_{n, 3}^{(a)}(r)\right), \\
& \mathbf{F}_{n}^{(b)}(r)=\left(\mathbf{f}_{n, 1}^{(b)}(r) \mathbf{f}_{n, 2}^{(b)}(r) \mathbf{f}_{n, 3}^{(b)}(r)\right) .
\end{aligned}
$$

В зависимости от значения потенциала примеси на данном отрезке, среди этих решений могут быть бегущие волны, а также экспоненциально растущие и убывающие функции. Бегущие волны можно поместить в любую группу ( $a$ или $b)$. Важно, чтобы экспоненциально убывающие решения оказались в группе $a$, а экспоненциально растущие - в группе $b$. Общее решение будет линейной комбинацией этих 6 элементарных решений, которую мы представим в виде произведения матрицы на векторстолбец коэффициентов:

$$
\mathbf{f}_{n}(r)=\left(\begin{array}{ll}
\mathbf{F}_{n}^{(a)}(r) & \mathbf{F}_{n}^{(b)}(r)
\end{array}\right)\left(\begin{array}{l}
\mathbf{a}_{n} \\
\mathbf{b}_{n}
\end{array}\right) .
$$

Матрицы рассеяния связывают коэффициенты на разных отрезках следующим образом:

$$
\left(\begin{array}{c}
\mathbf{a}_{n} \\
\mathbf{b}_{m}
\end{array}\right)=\left(\begin{array}{ll}
\mathbf{S}_{m n}^{(a a)} & \mathbf{S}_{m n}^{(a b)} \\
\mathbf{S}_{m n}^{(b a)} & \mathbf{S}_{m n}^{(b b)}
\end{array}\right)\left(\begin{array}{l}
\mathbf{a}_{m} \\
\mathbf{b}_{n}
\end{array}\right),
$$

где предполагается, что $n \leq m$. Формулы для вычисления этих матриц приведены в работе [11] и используют трансфер-матрицы для соседних отрезков:

$$
\left(\begin{array}{l}
\mathbf{a}_{n} \\
\mathbf{b}_{n}
\end{array}\right)=\left(\begin{array}{ll}
\mathbf{T}_{n}^{(a a)} & \mathbf{T}_{n}^{(a b)} \\
\mathbf{T}_{n}^{(b a)} & \mathbf{T}_{n}^{(b b)}
\end{array}\right)\left(\begin{array}{c}
\mathbf{a}_{n-1} \\
\mathbf{b}_{n-1}
\end{array}\right)
$$

Зная элементарные частные решения, трансфер-матрицы легко найти из условия непрерывности радиальной функции и ее первой производной на границах отрезков:

$$
\begin{aligned}
& \left(\begin{array}{cc}
\mathbf{T}_{n}^{(a a)} & \mathbf{T}_{n}^{(a b)} \\
\mathbf{T}_{n}^{(b a)} & \mathbf{T}_{n}^{(b b)}
\end{array}\right)=\left(\begin{array}{cc}
\mathbf{F}_{n}^{(a)}\left(r_{n}\right) & \mathbf{F}_{n}^{(b)}\left(r_{n}\right) \\
\mathbf{F}_{n}^{\prime(a)}\left(r_{n}\right) & \mathbf{F}_{n}^{\prime(b)}\left(r_{n}\right)
\end{array}\right)^{-1} \\
& \times\left(\begin{array}{cc}
\mathbf{F}_{n-1}^{(a)}\left(r_{n}\right) & \mathbf{F}_{n-1}^{(b)}\left(r_{n}\right) \\
\mathbf{F}_{n-1}^{\prime(a)}\left(r_{n}\right) & \mathbf{F}_{n-1}^{\prime(b)}\left(r_{n}\right)
\end{array}\right) .
\end{aligned}
$$

В качестве приближенных частных решений обычно используются решения для постоянного или полиномиального потенциала на отрезке $[13,14]$. Такой подход, 
однако, в случае сферических волн имеет существенный недостаток: все сферические функции Бесселя (кроме функций первого рода) имеют особенность при нулевом значении аргумента. Из-за этого некоторые частные решения вблизи примесного центра (при малых $r$ ) и точки поворота (при малых $k$ ) будут иметь слишком большую амплитуду.

В данной работе мы предлагаем использовать в качестве частных решений результат прямого интегрирования нашей системы методом Рунге-Кутта в пределах каждого отрезка со следующими начальными условиями:

$$
\begin{aligned}
& \mathbf{F}_{n}^{(a)}\left(r_{n+1}\right)=\mathbf{F}_{n}^{(b)}\left(r_{n}\right)=\left(\begin{array}{lll}
1 & 0 & 0 \\
0 & 1 & 0 \\
0 & 0 & 1
\end{array}\right), \\
& \mathbf{F}_{n}^{\prime(a)}\left(r_{n+1}\right)=\mathbf{F}_{n}^{\prime(b)}\left(r_{n}\right)=\left(\begin{array}{lll}
i & 0 & 0 \\
0 & i & 0 \\
0 & 0 & i
\end{array}\right) .
\end{aligned}
$$

Интегрирование для решения типа $a$ следует проводить в направлении убывания $r$ (от $r_{n+1}$ до $r_{n}$ ), а для решений типа $b-$ в направлении роста $r$ (от $r_{n}$ до $r_{n+1}$ ). За счет имеющейся в системе неустойчивости данный подход обеспечит необходимую структуру решений: убывание для функций типа $a$ и рост для функций типа $b$.

Отрезок от 0 до $r_{1}$ следует рассмотреть отдельно. Из-за особенности потенциала примеси и коэффициентов уравнения (4) вблизи начала координат здесь невозможно провести интегрирование уравнения обычным способом. Для того чтобы получить решение вблизи нуля, нужно использовать обобщенный метод Фробениуса [17]. В нашем случае применение этого метода дает начальные условия для трех линейно независимых решений, не имеющих особенностей вблизи примесного центра. Без ограничения общности мы можем считать их решениями типа $a$, и положить

$$
\begin{gathered}
\mathbf{b}_{0}=0, \\
\mathbf{F}_{0}^{(b)}(r)=0 .
\end{gathered}
$$

Тогда трансфер-матрица в точке $r_{1}$ будет иметь вид

$$
\mathbf{T}_{1}=\left(\begin{array}{ll}
\mathbf{T}_{1}^{(a a)} & 0 \\
\mathbf{T}_{1}^{(b a)} & 0
\end{array}\right)
$$

Применяя формулы для вычисления матрицы рассеяния [11], мы получим следующее выражение:

$$
\mathbf{S}_{0, n}=\left(\begin{array}{cc}
\mathbf{S}_{1, n}^{(a a)} \mathbf{T}_{1}^{(a a)} & \mathbf{S}_{1, n}^{(a b)} \\
\mathbf{S}_{1, n}^{(b a)} \mathbf{T}_{1}^{(a a)}-\mathbf{T}_{1}^{(b a)} & \mathbf{S}_{1, n}^{(b b)}
\end{array}\right)
$$

Как будет показано далее, коэффициент $\mathbf{b}_{n}$ можно считать известным, и тогда мы сможем найти $\mathbf{a}_{0}$ с помощью следующего выражение, которое следует из определения матрицы рассеяния (11) в случае $\mathbf{b}_{0}=0$ :

$$
\mathbf{S}_{0, n}^{(b a)} \mathbf{a}_{0}=-\mathbf{S}_{0, n}^{(b b)} \mathbf{b}_{n}
$$

Зная все промежуточные матрицы рассеяния, а также коэффициенты $\mathbf{a}_{0}$ и $\mathbf{b}_{n}$, мы можем найти коэффициенты $\mathbf{a}_{m}$ и $\mathbf{b}_{m}$ на каждом отрезке (подробнее см. работу [11]).

Для того чтобы определить значение $\mathbf{b}_{n}$, мы предположили, что при $r>r_{n}$ потенциал равен нулю, где минимальная допустимая величина $r_{n}$ определяется концентрацией примесей. Мы рассматриваем каждую примесь независимо от $r$ других, и поэтому можно считать, что отдельный центр не действует на электрон за пределами выделенного ему объема, который имеет порядок $r_{n}^{3}$. Концентрация примесей, соответственно, будет иметь порядок величины $r_{n}^{-3}$. В наших расчетах мы считали потенциал нулевым за пределами сферы радиуса $r_{n}=100$ нм. Соответствующая концентрация носителей составляет $10^{15} \mathrm{~cm}^{-3}$, что является характерным значением для нелегированных образцов.

В запрещенной зоне, т. е. в области дискретного спектра, при $r>r_{n}$ все решения типа $b$ будут растущими экспонентами, и поэтому следует положить

$$
\mathbf{b}_{n}=0 \text {. }
$$

Тогда мы получаем следующее уравнение для $\mathbf{a}_{0}$ :

$$
\mathbf{S}_{0, n}^{(b a)} \mathbf{a}_{0}=0
$$

которое, очевидно, имеет ненулевые решения только при условии

$$
\operatorname{det} \mathbf{S}_{0, n}^{(b a)}(E)=0 .
$$

Так как матрица рассеяния зависит от энергии состояния, исходя из данного условия можно получить энергии состояний дискретного спектра.

В зоне проводимости при $r>r_{n}$ существует по одному решению $a$ и $b$ типа для бегущих волн и по два решения для растущих и убывающих экспонент. Исходя из граничных условий, на бесконечности не должно быть растущих экспонент. Будем считать, что решение в виде бегущей волны является первым столбцом в матрице (10). Тогда мы можем положить

$$
\mathbf{b}_{n}=\left(\begin{array}{l}
1 \\
0 \\
0
\end{array}\right)
$$

В таком случае коэффициент $\mathbf{a}_{0}$ вычисляется по формуле

$$
\mathbf{a}_{0}=-\left(\mathbf{S}_{0, n}^{(b a)}\right)^{-1} \mathbf{S}_{0, n}^{(b b)} \mathbf{b}_{n} .
$$

Для резонансных состояний характерна большая амплитуда волновой функции вблизи примесного центра, т.е. большие по модулю компоненты вектора $\mathbf{a}_{0}$ по сравнению с $\mathbf{b}_{n}$. Из выражения (12) следует, что это 

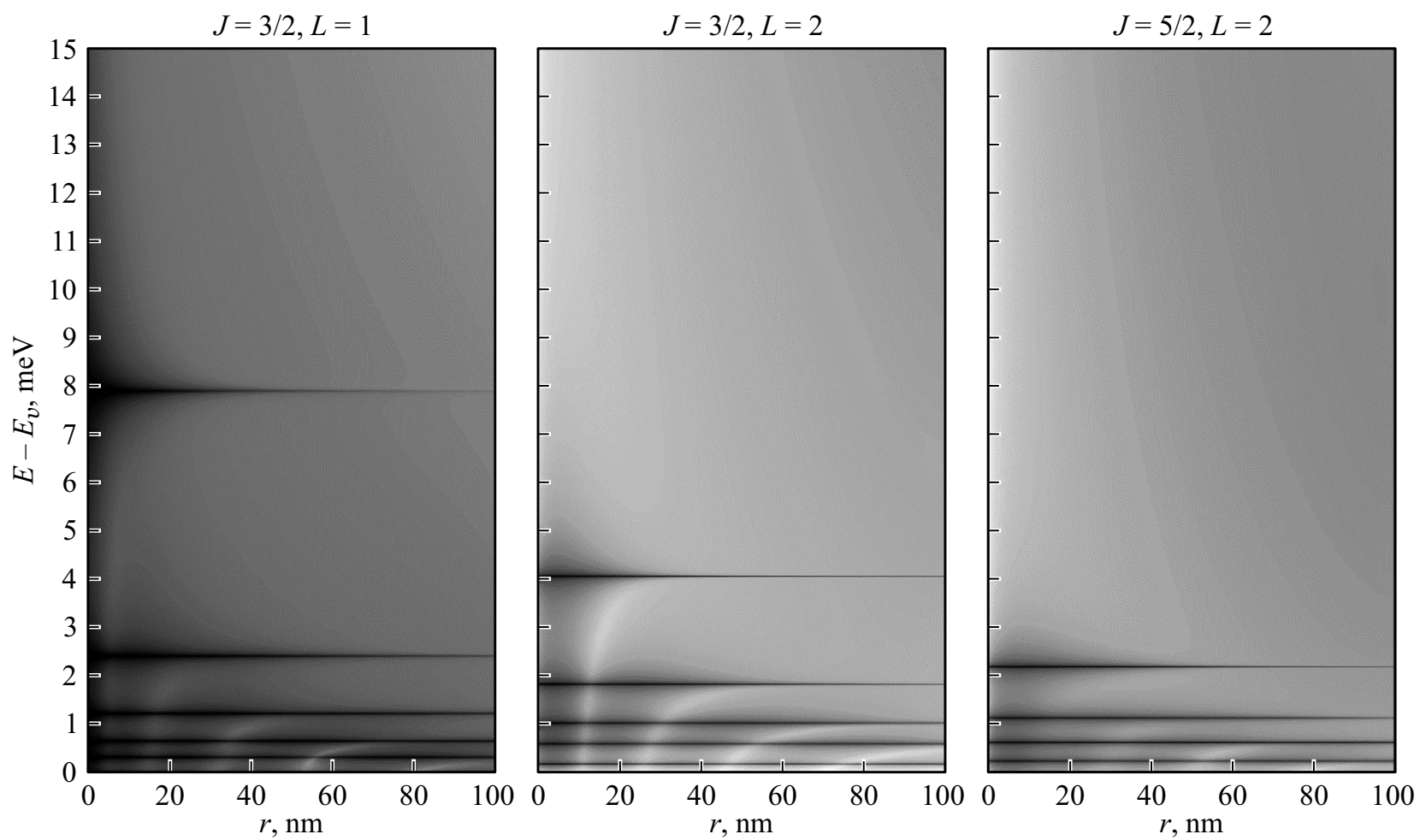

Рис. 2. Зависимость логарифма плотности вероятности обнаружения электрона с определенной энергией $E$ на определенном расстоянии $r$ от кулоновского акцептора в объемном $\mathrm{Hg}_{0.84} \mathrm{Cd}_{0.16}$ Те. Данная величина вычислена как квадрат модуля вектора $\mathbf{f}_{E}(r)$, т. е. усреднена по углам $\theta$ и $\vartheta$. Темный цвет соответствует бо́льшим значениям плотности вероятности.

достигается, когда матрица $\mathbf{S}_{0, n}^{(b a)}(E)$ имеет определитель по модулю, близкий к нулю. Для дискретных уровней, как было показано выше, он в точности равен нулю. Таким образом, условием для определения положения как дискретных, так и резонансных примесных состояний являются максимумы следующей функции энергии:

$$
R(E)=\left|\operatorname{det} \mathbf{S}_{0, n}^{(b a)}(E)\right|^{-1} .
$$

При численных расчетах определитель в этой формуле, как правило, не обращается в нуль даже в дискретном спектре из-за ограничений в точности.

\section{3. Результаты и обсуждение}

Для расчета примесных состояний в КРТ мы использовали те же параметры материалов, что и в работе [8]. Чтобы учесть зависимость ширины запрещенной зоны $\left(E_{g}\right)$ от состава твердого раствора $(x)$ и температуры $(T)$, мы воспользовались следующей формулой [18]:

$$
\begin{aligned}
E_{g}(x, T)= & -303(1-x)+1606 x-132 x(1-x) \\
& -(-0.63(1-x)+0.325 x+0.592 x(1-x)) \\
& \times \frac{T^{2}}{11(1-x)+78.7 x+T},
\end{aligned}
$$

где энергия выражена в мэВ, а температура — в К.
Мы провели расчеты для твердого раствора КРТ с $x=0.16$. Этот случай представляет наибольший интерес, так как в данном материале зонная структура меняется от инвертированной к нормальной при увеличении температуры. Следует ожидать, что при низких температурах у акцептора в данном материале будут только резонансные состояния. При открытии энергетической щели с ростом температуры часть состояний станут локализованными, а самые глубокие останутся резонансными. При дальнейшем увеличении температуры все уровни будут дискретными.

В данной работе мы не будем рассматривать состояния с $J=1 / 2$, так как их энергия зависит прежде всего от массы легких дырок, которая в узкозонных материалах очень мала. Известно, что наибольшей глубиной обладает состояние с $J=3 / 2, L=1[8,9]$. Кроме этих состояний, мы рассмотрим также уровни с $J=3 / 2$, $L=2$ и $J=5 / 2, L=2$. При больших значениях углового момента возникают технические сложности из-за малой амплитуды волновой функции вблизи примесного центра. Так как поиск таких решений выходит за рамки данной работы, просто отметим, что эту проблему можно обойти с помощью следующей замены неизвестных функций в уравнении (4):

$$
\mathbf{f}(r)=r^{L-1} \mathbf{g}(r) .
$$

Результаты расчетов волновых функций состояний непрерывного спектра при нулевой температуре приведены на рис. 2. Как уже отмечалось ранее, в данных 

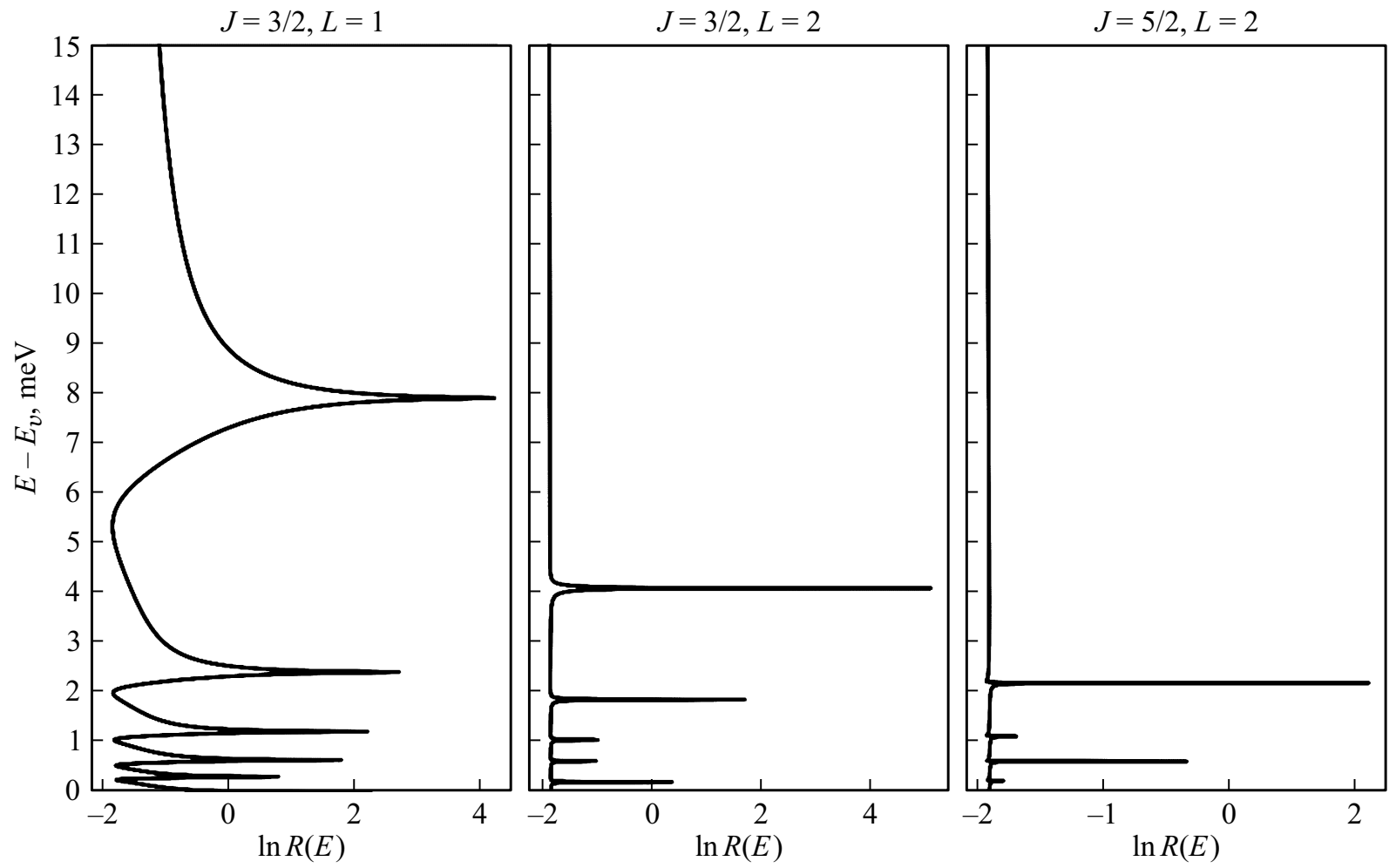

Рис. 3. Логарифм функции $R(E)$ для объемного $\mathrm{Hg}_{0.84} \mathrm{Cd}_{0.16} \mathrm{Te}$.
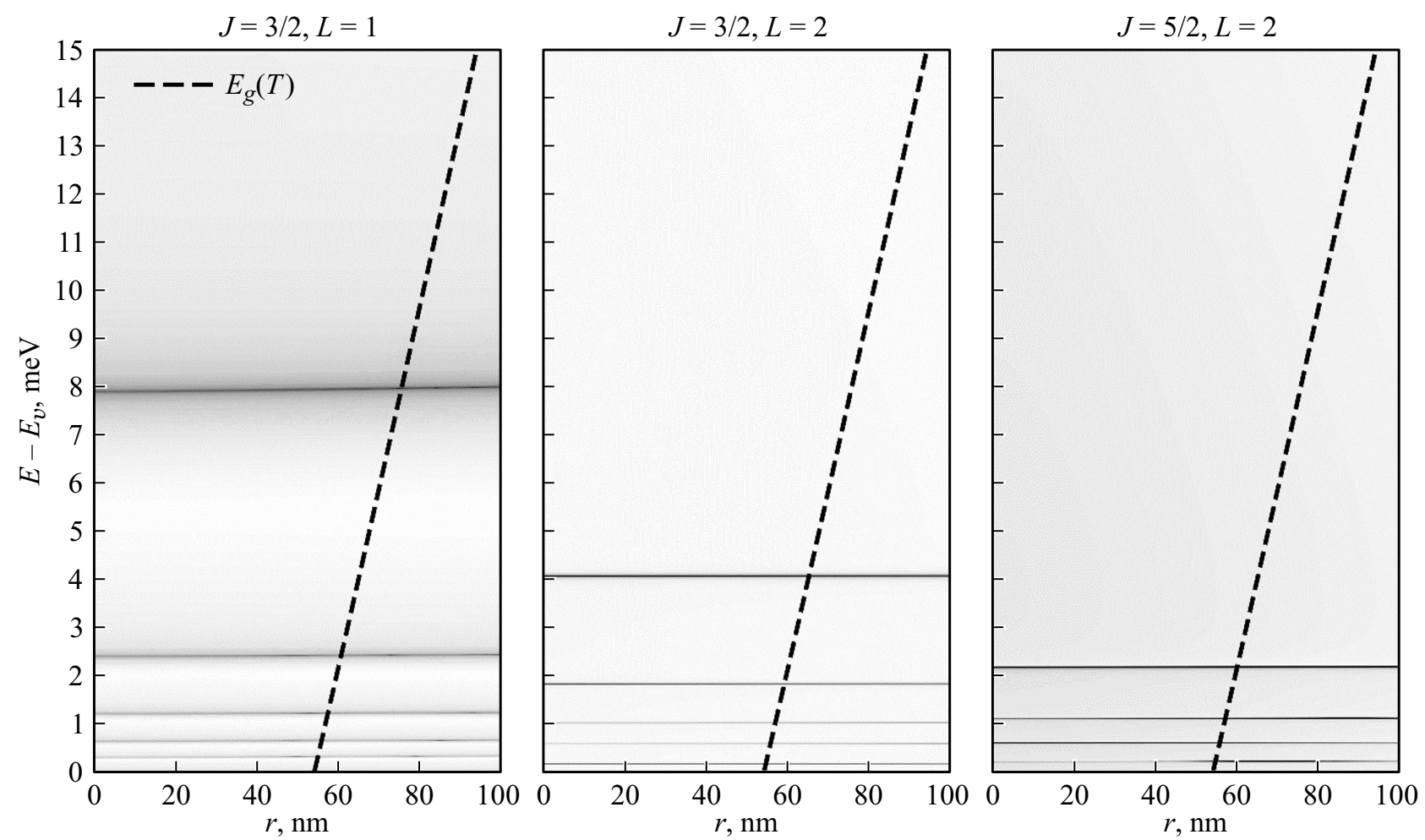

Рис. 4. Зависимость логарифма функции $R(E)$ от температуры для объемного $\operatorname{Hg}_{0.84} \mathrm{Cd}_{0.16}$ Те. Темный цвет соответствует бо́льшим значениям, светлый - меньшим. 
условиях все состояния акцептора являются резонансными. Они хорошо видны как сгущения плотности вероятности вблизи примеси при некоторых значениях энергии. У уровней с меньшей энергией можно заметить минимумы, которые соответствуют узлам волновой функции возбужденных состояний в дискретном спектре. График $R(E)$ для нулевой температуры приведен на рис. 3. Легко заметить, что положение резонансных уровней точно соответствует максимумам этой функции.

Энергии состояний при разных значениях температуры можно легко определить из графика функции $R(E)$ для всех температур, приведенного на рис. 4. Темные линии - это максимумы данной функции, которые соответствуют энергии резонансных или локализованных уровней. Хорошо видно, что данный метод одинаково применим как в области непрерывного спектра, так и в запрещенной зоне.

Легко видеть, что энергии примесных состояний практически не изменяются с ростом температуры, несмотря на существенное изменение положения зоны проводимости, а значит, массы легких дырок и электронов. Данный факт хорошо согласуется с результатами экспериментальных наблюдений [7] и подтверждает выводы, относительно примесной природы наблюдавшихся в этой работе спектральных линий, сделанные на основе моделирования без непосредственного учета зоны проводимости. Изменение положения этой зоны под влиянием температуры не влияет на глубокие акцепторные состояния даже если они находятся внутри нее, так как они связаны прежде всего с зоной тяжелых дырок.

\section{4. Заключение}

В данной работе предложен универсальный метод вычисления энергии примесных состояний, который применим как для локализованных, так и для резонансных уровней. Данный подход основан на методе матрицы рассеяния и потому обладает устойчивостью, что позволяет применять его в многозонных моделях. Таким образом, он очень удобен для описания узкозонных полупроводниковых структур.

Теоретически доказано, что энергии акцепторных состояний в узкозонном твердом растворе $\mathrm{Hg}_{1-x} \mathrm{Cd}_{x} \mathrm{Te}$ практически постоянны в широком диапазоне температур (от 0 до $100 \mathrm{~K}$ ), несмотря на существенное изменение положения зоны проводимости, а также массы электронов и легких дырок. Такое поведение имеет место в том числе для резонансных уровней, расположенных непосредственно в зоне проводимости.

\section{Финансирование работы}

Разработка программного обеспечения для расчета температурной зависимости волновых функций и энергий примесных состояний выполнена при поддержке Российского научного фонда (грант № 19-72-00128). Анализ результатов расчета волновых функций с определением энергий примесных состояний выполнен при поддержке Министерства образования и науки Российской Федерации (проект МК-1430.2020.2).

\section{Конфликт интересов}

Авторы заявляют, что у них нет конфликта интересов.

\section{Список литературы}

[1] E.G. Novik, A. Pfeuffer-Jeschke, T. Jungwirth, V. Latussek, C.R. Becker, G. Landwehr, H. Buhmann, L.W. Molenkamp. Phys. Rev. B, 72, 035321 (2005).

[2] N.N. Mikhailov, R.N. Smirnov, S.A. Dvoretsky, Yu.G. Sidorov, V.A. Shvets, E.V. Spesivtsev, S.V. Rykhlitski. IJNT, 3, 120 (2006).

[3] S. Dvoretsky, N. Mikhailov, Y. Sidorov, V. Shvets, S. Danilov, B. Wittman, S. Ganichev. J. Electron. Mater., 39, 918 (2010).

[4] S.V. Morozov, V.V. Rumyantsev, M.A. Fadeev, M.S. Zholudev, K.E. Kudryavtsev, A.V. Antonov, A.M. Kadykov, A.A. Dubinov, N.N. Mikhailov, S.A. Dvoretsky, V.I. Gavrilenko. Appl. Phys. Lett., 111, 192101 (2017).

[5] Д.В. Козлов, В.В. Румянцев, С.В. Морозов, А.М. Кадыков, М.A. Фадеев, Н.-W. Hubers, В.И. Гавриленко. ФТП, 52, 1257 (2018).

[6] Д.В. Козлов, В.В. Румянцев, А.М. Кадыков, М.А. Фадеев, Н.С. Куликов, В.В. Уточкин, Н.Н. Михайлов, С.А. Дворецкий, В.И. Гавриленко, Х.-В. Хюберс, Ф. Теппе, С.В. Морозов. Письма ЖЭТФ, 109, 679 (2019).

[7] Д.В. Козлов, В.В. Румянцев, С.В. Морозов, А.М. Кадыков, В.С. Варавин, Н.Н. Михайлов, С.А. Дворецкий, В.И. Гавриленко, F. Терре. ФТП, 49, 1654 (2015).

[8] М.С. Жолудев, Д.В. Козлов, Н.С. Куликов, А.А. Разова, В.И. Гавриленко, С.В. Морозов. ФТП, 54, 695 (2020).

[9] A. Baldereschi, N.O. Lipari. Phys. Rev. B, 8, 2697 (1973).

[10] E.P. Pokatilov, V.A. Fonoberov, V.M. Fomin, J.T. Devreese. Phys. Rev. B, 64, 245328 (2001).

[11] D.Y.K. Ko, J.C. Inkson. Phys. Rev. B, 38, 9945 (1988).

[12] M.S.D. Bianchi, M.D. Ventra. Eur. J. Phys., 16, 260 (1995).

[13] C. Ramírez, R. León. J. Phys. Soc. Jpn., 86, 114002 (2017).

[14] C. Ramírez, F.H. González, C.G. Galván. J. Phys. Soc. Jpn., 88, 094002 (2019).

[15] С.В. Морозов, М.С. Жолудев. Письма ЖТФ, 47, 26 (2021).

[16] М.С. Жолудев, В.В. Румянцев, С.В. Морозов. ФТП, 55, 391 (2021).

[17] M. Barkatou, T. Cluzeau, C. El Bacha. In: Proc. of Mathematical Theory of Networks and Systems (Budapest, Hungary, 2010) p. 1059.

[18] J.P. Laurenti, J. Camassel, A. Bouhemadou, B. Toulouse, R. Legros, A. Lusson. J. Appl. Phys., 67, 6454 (1990).

Редактор А.Н. Смирнов 


\title{
Calculation of temperature dependence of coulomb acceptor state energy in narrow-gap $\mathrm{HgCdTe}$
}

\author{
M.S. Zholudev ${ }^{1,2}$, V.V. Rumyantsev ${ }^{1,2}$, S.V. Morozov ${ }^{1,2}$ \\ ${ }^{1}$ Institute for Physics of Microstructures, \\ Russian Academy of Sciences, \\ 603950 Nizhny Novgorod, Russia \\ ${ }^{2}$ Lobachevsky State University of Nizhny Novgorod, \\ 603950 Nizhny Novgorod, Russia
}

\begin{abstract}
Localized and resonant state energies of coulomb acceptor in narrow-gap HgCdTe alloy are calculated at different temperatures. A universal technique for estimation of localized and resonant state energies is suggested. It is shown theoretically that these energies are constant in a wide range of temperatures from 0 to $100 \mathrm{~K}$. The simulation is performed with scattering matrix method within spherically-symmetric three-band Kane model that takes into account the conduction band two valence bands.
\end{abstract}

